\title{
Hybrid Monolith of Graphene/TEMPO-Oxidized Cellulose Nanofiber as Mechanically Robust, Highly Functional, and Recyclable Adsorbent of Methylene Blue Dye
}

\author{
Asif Hussain, Jiebing Li, Jun Wang, Fei Xue, Yundan Chen, \\ Tallal Bin Aftab, and Dengxin Li \\ College of Environmental Science and Engineering, Donghua University, Shanghai 201620, China \\ Correspondence should be addressed to Dengxin Li; lidengxin@mail.dhu.edu.cn
}

Received 10 September 2017; Accepted 2 November 2017; Published 3 January 2018

Academic Editor: Sónia Simões

Copyright (C) 2018 Asif Hussain et al. This is an open access article distributed under the Creative Commons Attribution License, which permits unrestricted use, distribution, and reproduction in any medium, provided the original work is properly cited.

Herein we demonstrate first report on fabrication, characterization, and adsorptive appraisal of graphene/cellulose nanofibers (GO/CNFs) monolith for methylene blue (MB) dye. Series of hybrid monolith (GO/CNFs) were assembled via urea assisted selfassembly method. Hybrid materials were characterized by scanning electron microscopy, Fourier transform infrared spectroscopy, $\mathrm{X}$-ray diffraction patterns, Raman spectroscopy, elemental analysis, thermogravimetric curve analysis, specific surface area, surface charge density measurement, and compressional mechanical analysis. It was proposed that strong chemical interaction (mainly hydrogen bonding) was responsible for the formation of hybrid assembly. GO/CNFs monolith showed mechanically robust architecture with tunable pore structure and surface properties. GO/CNFs adsorbent could completely remove trace to moderate concentrations of $\mathrm{MB}$ dye and follow pseudo-second-order kinetics model. Adsorption isotherm behaviors were found in the following order: Langmuir isotherm > Freundlich isotherm > Temkin isotherm model. Maximum adsorption capacity of $227.27 \mathrm{mg} \mathrm{g}^{-1}$ was achieved which is much higher than reported graphene based monoliths and magnetic adsorbent. Incorporation of nanocellulose follows exponential relationship with dye uptake capacities. High surface charge density and specific surface area were main dye adsorptive mechanism. Regeneration and recycling efficiency was achieved up to four consecutive cycles with costeffective recollection and zero recontamination of treated water.

\section{Introduction}

The development of competent and eco-friendly adsorbent material for water decontamination has been an ever-growing area for academia and industry, in particular aromatic organic dyes, which possess many hazards such as toxic [1], nonbiodegradable [2], aesthetically objectionable, and light restrictive nature in aqueous environment $[1,3]$. Among the various treatment techniques such as biological treatment [4], ozone oxidation [4], catalytic degradation [5], photocatalytic oxidation [6], coagulation and flocculation [3], nanofiltration membrane [7], and unsaturated polyester resins [8], adsorption is the most attractive due to competency for variety of organic dyes, insensitivity to toxic pollutants [9], efficiency for low concentration range [10], easiness of operating, and being economical process $[9,11-21]$. Carbon materials in the form of activated carbon are historically dominant for dye decontamination in view of chemical stability, high surface area, and functional active sites [16, 20, 22-26]. Recently, graphene oxide (GO) produced by soft chemistry techniques arouse as highly efficient $[10,15,21,27-29]$ and low cost carbon nanoadsorbent $[11,30]$ followed by various carbon materials including single/or multiwall carbon nanotubes, since it owns many superior properties such as large theoretical surface area $\left(2630 \mathrm{~m}^{2} / \mathrm{g}\right)$ [31], mechanical flexibility, high charge carrier mobility, and chemical stability [32]. Apart from high efficiency, simple and economical recollection cannot be overlooked as well, in view of recontamination through introduction of nanohazard after treatment. So, exploring the eco-friendly formulation couple with high efficiency is of great concern and widely open field. 
Unique structural features of GO, which is composed of planner $\mathrm{sp}^{2}$ type random aromatic domains and $\mathrm{sp}^{3}$ hybridized sites containing plenty of oxygen functional groups (epoxy, hydroxyl, and carboxyl groups), make this nanomaterial amphiphilic. For example, oxygen functionality imparts high hydrophilicity, while aromatic domain renders GO sheets hydrophobic character [30]. Likewise, oxygen functional groups make this nanomaterial behave like polymer and form variety of covalent or noncovalent bonding reactions [32]. Recently, by taking advantage of fundamental chemistry of graphene oxide a diverse range of functional adsorbents have been formulated, for instance, chemically reduced graphene [27], sponge [12, 31], hydrogels/aerogels $[18,33]$, and magnetically separable adsorbents [9, 19, 34-37] to improve its competency and recollection issues. However, it suffers from low structural and adsorption capacities, while use of external magnate is practically not feasible as well. It has been reported that the affinity of dye pollutants toward graphene based adsorbent is attributed to active functional sites and pure aromatic domains or combination of them $[9,10,12-15,18,19,21,26,27,29,31,33-36,38-$ 40]. The former one can bind hydrophilic organic species via noncovalent forces such as vender wall and electrostatic and hydrogen bond interaction, whereas later one forms strong $\pi-\pi$ staking/or hydrophobic interaction with benzene ring of organic compounds. It is also widely accepted that porous material can considerably improve the dye capturing ability due to improving surface area. It means that strong interaction of certain pollutant molecules could significantly be improved through modification of structural, surface, and electronic flexibility of graphene. In this regard, self-assembly of graphene (GO) offers simple and scalable approach to integrate the superior properties of $2 \mathrm{D}$ nanobuilding blocks into interconnected 3D monolithic materials [41]. The interconnected pores arising from nanosize $2 \mathrm{D}$ sheets provide unique physicochemical properties such as microstructure, charge mobility, large surface area, and surface functionality depending on the preparation conditions and reacting precursors $[32,42]$. So far, different polymers/or molecules, for example, chitosan [43], resol [44], polymer [45, 46], carbohydrate [43], PVA [47], divalent metal ions [41], carbon nanotube [33], DNA [48], thiourea [31], aqueous ammonia solution [49], luffa sponge [12], and phenolic acid [50], have been reported for the assembly of macroscopic graphene by various physical or chemical interactions. However, very little work was reported for water decontamination applications. Most of the reported work is either selective for oil removal due to highly hydrophobic nature [49] or suffering from mechanically fragile three-dimensional structures and low adsorption capacity $[12,15,18,31,33,51]$ due to insufficient functional active sites and large pore size which considerably limit its easy regeneration-recycling properties, thus being not feasible for industrial applications.

Nanocellulose biopolymer recently arose as an attractive carbon material due to low cost and being biodegradable and green in nature $[52,53]$. Among the various methods, the production of nanocellulose fibers through 2,2,6,6-tetramethyl1-piperidinyloxy (TEMPO) mediated selective oxidation [5456] offers the nanolevel dispersibility of individual fibrils in pure aqueous medium, since it possesses high density of sodium carboxylated moieties on the fibril surfaces [55, 56]. Moreover, excellent mechanical strength (140 Mpa) and highly reactive moieties $\left(\mathrm{COONa}^{+}\right)$of this nanomaterial allow one to produce variety of mechanically robust functional composites [52, 53] including hydro/aerogels [57]. Inspired by the intrinsic properties of graphene oxide and nanocellulose fibrils, incorporation of these nanobuilding blocks during self-assembly technique could be a promising approach in order to optimize physicochemical properties and functionality of interconnected porous monolith for industrial application, since graphene can form variety of bonding interactions with this nanofiller such as electrostatic repulsion/attraction and hydrogen bonding. To our knowledge, self-assembly phenomena between graphene oxide and TEMPO-oxidized nanocellulose fibers hitherto are not practiced for the fabrication of monolith material.

In this study, herein we report on fabrication, characterization, and adsorptive evaluation of graphene/cellulose nanofibers (GO/CNFs) monolith for methylene blue (MB) dye. Series of hybrid monoliths (GO/CNFs) were assembled via urea assisted self-assembly method. Monolith was characterized by scanning electron microscopy (SEM), Fourier transform infrared spectroscopy (FTIR), X-ray diffraction (XRD), Raman spectroscopy, elemental analysis, thermogravimetric curve analysis (TGA), specific surface area (SSA), surface charge density measurement, and compressional mechanical analysis. Adsorption behaviors of dye were examined by equilibrium adsorption isotherms model (Langmuir, Freundlich, and Temkin isotherm) and kinetic model (pseudo-first kinetics and second-order kinetics). Statistical relationship between adsorption capacity and incorporated nanocellulose concentration was established. Regeneration and recycling experiment were performed for four consecutive cycles. Moreover, comparison of MB adsorption capacity with previously reported works was discussed here.

\section{Experimental Section}

2.1. Material. Natural flakes of graphite (500 mesh) were provided by Shanghai Yifan Graphite Co. Ltd. (China). Potassium permanganate (KMnO4), sodium nitrate (NaNO3), hydrogen peroxide ( $\mathrm{H} 2 \mathrm{O} 2,30 \%)$, sodium hydroxide $(\mathrm{NaOH})$, sulfuric acid (H2SO4, 98\%), microcrystalline cellulose (MCC), sodium hypochlorite solution ( $\mathrm{NaClO}$, $9 \%)$, sodium bromide $(\mathrm{NaBr})$, carbamide, methylene blue dye (C16H18ClN3S, MW: $373.9 \mathrm{~g} / \mathrm{mol}$ ), and 2,2,6,6tetramethylpiperidine-1-oxyl (TEMPO) were supplied by Sinopharm Chemical Reagent Co. Ltd. (China) and used without any further purification.

2.2. Preparation of Graphene Oxide. Graphene oxide (GO) was prepared with slight modification of published work about Hummers and Offeman method [58]. Briefly, $5 \mathrm{~g}$ of graphite powder was treated with sodium nitrate $(2.5 \mathrm{~g})$ and sulfuric acid ( $115 \mathrm{ml}, 98 \%)$; then oxidation was initiated by slow addition of potassium permanganate $(15 \mathrm{~g})$ on ice bath $\left(4^{\circ} \mathrm{C}\right)$ under constant stirring for $2 \mathrm{~h}$. After that, obtained slurry was set on water bath $\left(35^{\circ} \mathrm{C}, 2 \mathrm{~h}\right)$ under stirring; then 


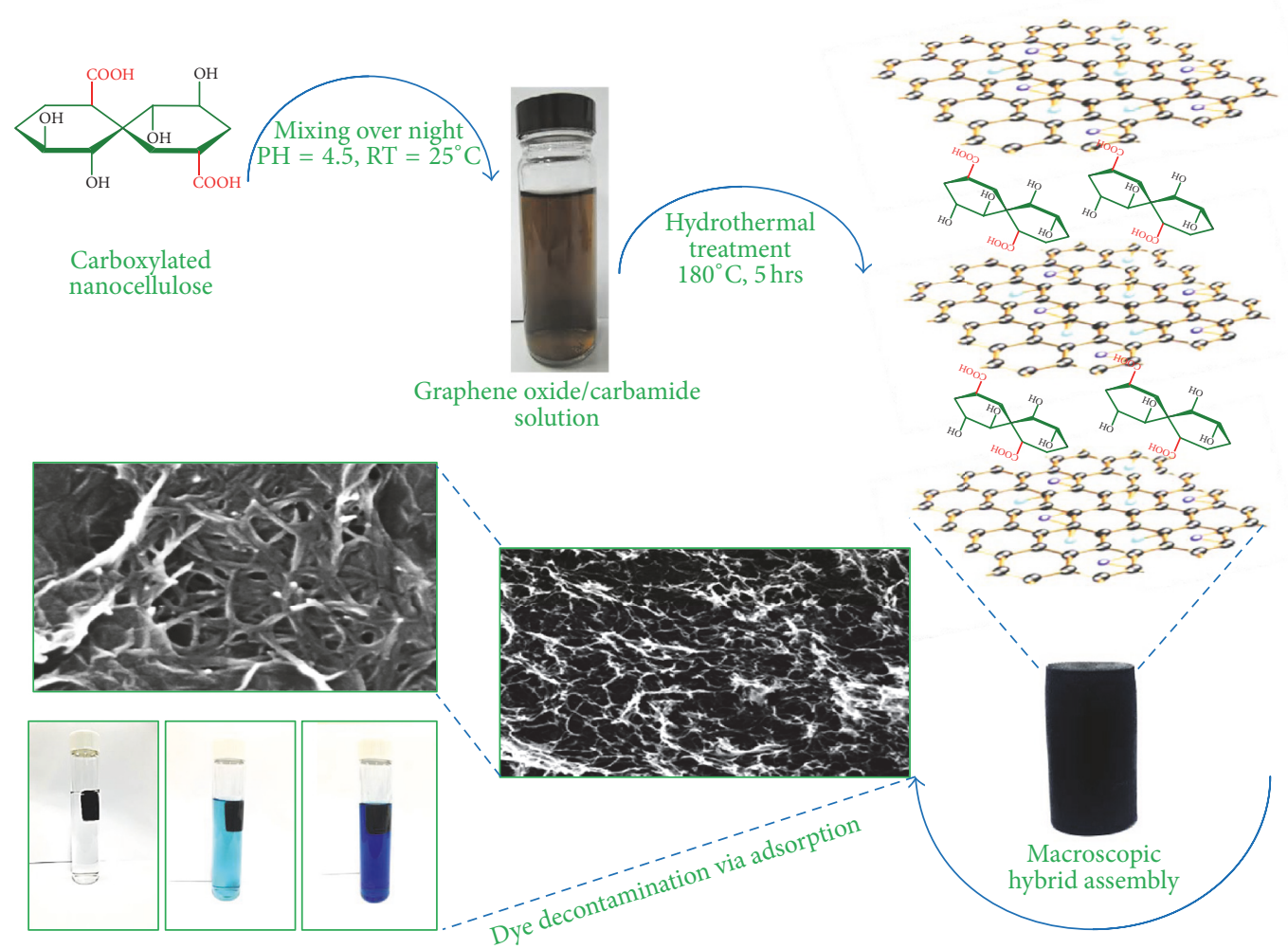

Scheme 1: Graphical illustration of GO/CNFs monolith assembly.

$230 \mathrm{ml}$ of distilled water was added and kept at $98^{\circ} \mathrm{C}$ for further $15 \mathrm{~min}$. After completing the oxidation, the reaction was quenched by adding $700 \mathrm{ml}$ of distilled water followed by hydrogen peroxide $(30 \%, 50 \mathrm{ml})$. Upon quenching, the color of solution changes from dark brown to bright yellow. The product was centrifuged and purified via washing with $\mathrm{HCl}$ $(1: 10,500 \mathrm{ml})$ followed by plenty of deionized water until $\mathrm{pH}$ $=6$. Finally, the product was subjected to freeze-drying and labeled as GO for further use.

2.3. Preparation of Carboxylated Cellulose Nanofibers. The primary hydroxyl groups on the surface of fibrous microcrystalline cellulose were selectively oxidized to sodium carboxylate groups (COONa+) using TEMPO/NaOCl/NaBr system according to previous methods $[54,55]$ and stored at $4^{\circ} \mathrm{C}$ for further use (see ESI Section 1). To prepare carboxylated nanocellulose fibers (CNFs), suspension of sodium carboxylate cellulose fibers (TOCNFs) was mechanically homogenized ( $8000 \mathrm{rpm}, 10 \mathrm{~min}$ ) for further defibrillation and centrifuged $(12000 \mathrm{~g}, 10 \mathrm{~min})$ for the removal of partly fibrillated fraction. Then, never dry TOCNFs were diluted using ultrapure water $(0.1 \%)$ and treated with HCL $(1 \mathrm{M})$ for 3 hours for ion exchange and conversion of sodium carboxylated type $\mathrm{CNF}-\mathrm{COONa}+$ to $\mathrm{CNF}-\mathrm{COOH}+$. The product was washed with water until $\mathrm{pH}=4.0$ and labeled as CNFs. Finally, desired concentration of suspension $\left(16 \mathrm{mg} \mathrm{ml}^{-1}\right)$ was obtained and stored at 4 Co for further experimental work. The concentration of CNFs was calculated by the weight difference of CNFs suspension $(10 \mathrm{ml})$ after freezedrying. The carboxyl content of nanofibers $(0.547 \mathrm{mmol} / \mathrm{g})$ was estimated according to ESI Section 1. The successful conversion was confirmed by the appearance of new peak at $1732 \mathrm{~cm}^{-1}$ in FTIR spectra (Figure S1).

2.4. Fabrication of GO/CNFs Hybrid Monolith. To fabricate the GO/CNFs porous monolith, first, well-dispersed suspension of freeze-dried graphene oxide $\left(2 \mathrm{mg} \mathrm{ml}^{-1}, 200 \mathrm{ml}\right)$ was obtained by tip sonication in ultrapure water. Secondly, fixed amount of carbamide salt ( $800 \mathrm{mg}$ ) was mixed with graphene solution ( $\mathrm{pH}=4.5,1 \mathrm{~h})$; then known volume $(x=0,1,3,5$, and $7 \mathrm{ml}$ ) of separately prepared carboxylate cellulose nanofibers (CNFs) suspension was added to graphene/carbamide suspension followed by stirring overnight to obtain the homogenous solution. After that, $25 \mathrm{ml}$ of mixture was sealed in Teflon-lined stainless steel autoclave $(50 \mathrm{ml})$ and placed at 180 Co for hydrothermal treatment (5 hours). After cooling the reactor naturally at room temperature, cylindrical shape monolith was obtained (see graphical Scheme 1). The monolith was dipped into distilled water to remove residual carbamide and subjected to vacuumed freeze-drying (48 h). Product was labeled according to incorporated nanocellulose content as GO/CNF0, GO/CNF1, GO/CNF3, GO/CNF5, and $\mathrm{GO} / \mathrm{CNF}$, respectively, and used for further characterization and dye adsorption studies (see detail in ESI Section 2). 
2.5. Material Characterization. Morphology and microstructure of monolith were analyzed by scanning electron microscopy (SEM-S420, HITACHI) equipped with a field-emission electron gun. The Fourier transform infrared (FTIR) spectra were recorded over the range of $400-4000 \mathrm{~cm}^{-1}$ using $\mathrm{KBr}$ pressed disc method (NICOLET-6700, UK). Raman spectra were recorded via Renishaw Raman spectrophotometer (NEXUS-670, USA). The percentage of carbon (\% C), hydrogen $(\% \mathrm{H})$, and nitrogen $(\% \mathrm{~N})$ contents were determined by element analyzer (ELMENTAR, VAIRO EL-III, Germany). X-ray diffraction (XRD) patterns were recorded by X-ray diffractometer (RIGAKU, D/Max-2550pc) at operating voltage of $40 \mathrm{kV}$, current density $200 \mathrm{~mA}$, and $2 \theta$ scanning rage of $10^{\circ}-70^{\circ}$ using $\mathrm{Cu} \mathrm{K} \alpha$ radiation. Thermal stability of materials was measured by thermogravimetric analyzer (NETZSCH, TG-209F1, Germany) under nitrogen environment and temperature range of $30-900^{\circ} \mathrm{C}$ using heating rate of $10^{\circ} \mathrm{C} \mathrm{min}^{-1}$. Compression property of monolith was measured by dynamic mechanical analyzer (HY-940FS, China). Zeta potential measurement (ZP) was carried out by ZETASIZER (Nano-ZS90). Specific surface areas (SSA) of samples were calculated by the following formula [18]:

$$
\mathrm{SSA}=\frac{N_{\mathrm{A}} A_{\mathrm{MB}}\left(C_{o}-C_{e}\right) V}{M_{\mathrm{MB}} M_{\mathrm{S}}},
$$

where $N_{\mathrm{A}}$ is Avogadro's number $\left(6.023 \times 1023 \mathrm{~mol}^{-1}\right), A_{\mathrm{MB}}$ is the covered area per $\mathrm{MB}$ molecules (typically assumed to be $1.35 \mathrm{~nm}^{2}$ ), $C_{o}$ and $C_{e}$ are the initial and equilibrium concentration of $\mathrm{MB}$, respectively, $V$ is the volume of $\mathrm{MB}$ solution, $M_{\mathrm{MB}}$ is the relative molecular mass of $\mathrm{MB}$, and $M_{\mathrm{S}}$ is the mass of sample.

2.6. Dye Adsorption Studies. Aqueous solutions of methylene blue $(\mathrm{MB})$ dye with different concentration (10-100, $150,200,250$, and $300 \mathrm{mg} \mathrm{L}^{-1}$ ) were prepared by successive dilution of stock solution $\left(1000 \mathrm{mg} \mathrm{L}^{-1}\right)$ in deionized water. The residual MB concentration was measured by UVspectrophotometer (Shimadzu UV-spectrophotometer, UV1800) using an absorbance at $\lambda=664 \mathrm{~nm}$. Dye adsorption spectra were recorded over the range 200-800 nm using UVVIS-NIR spectrophotometers (UV-3600, SHIMADZU). Dye adsorption quantity $\left(\mathrm{mgg}^{-1}\right)$ and percentage removal (\%) were calculated by (2) and (3), respectively:

$$
\begin{aligned}
Q_{e} & =\frac{\left(C_{o}-C_{e}\right) V}{W}, \\
\operatorname{Removal}(\%) & =\frac{\left(C_{o}-C_{t}\right)}{C_{o}} \times 100,
\end{aligned}
$$

where $C_{o}\left(\mathrm{mg} \mathrm{L}^{-1}\right)$ and $C_{t}\left(\mathrm{mg} \mathrm{L}^{-1}\right)$ represent the initial and final $(t)$ time concentrations of dye in the solution. $V(\mathrm{~L})$ is the volume of the solution and $W(\mathrm{~g})$ is the mass of adsorbent.

\section{Results and Discussion}

3.1. Macroscopic and Microstructure Analysis. The macroscopic images of hybrid monolith have been presented in
Figure $\mathrm{S} 2(\mathrm{a}-\mathrm{d})$. It can be comprehended that all samples can easily be assembled into cylindrical shaped 3D material according to size and shape of the reaction vessel. To further examine the role of nanocellulose, the microstructure morphology of samples was investigated by SEM analysis. From Figure 1, all samples exhibited formation of interconnected 3D morphology and it can be noted that incorporation of CNFs with GO brought significant change in pore wall thickness and shrinkage in average size compared to those of GO/CNF0 (Figures 1(a), 1(c), 1(e), and 1(g)). High magnification SEM image of hybrid samples showed the presence of needle-like nanocellulose fibers in random direction which behave as compact skeleton within the dense and wrinkling type graphene sheets. Thus, it can be inferred that these surface decorated/adsorbed nanocellulose fibers may promote roughness between adjacent graphene sheets that results in compact $3 \mathrm{D}$ assembly due to strong chemical interaction (see FTIR section).

3.2. FTIR Analysis. To gain insight into the formation chemistry of hybrid monolith, FTIR spectra of samples have been presented in Figure 2. The successful oxidation of graphene oxide was confirmed by the presence of various kinds of oxygen functionality such as peak at 3422, 1715, 1628,1385 , and $1076 \mathrm{~cm}^{-1}$, which are mainly bloated from the starching vibration of hydroxyl group $(-\mathrm{OH})$, carboxylic group $(-\mathrm{C}=\mathrm{O})$, aromatic skeleton of graphitic domains $(\mathrm{C}=\mathrm{C})$ [52], and alkoxy (C-O) and epoxy group (C-O), respectively. Spectra of sodium carboxylated nanocellulose showed shifting of carboxyl band $(\mathrm{C}=\mathrm{O})$ from $1619 \mathrm{~cm}^{-1}$ to carboxylic starching $1732 \mathrm{~cm}^{-1}$ which confirmed the successful oxidation and conversion of sodium carboxylated into free carboxylated group after acid treatment. The spectra of carboxylated nanocellulose show characteristic bands at $1430 \mathrm{~cm}^{-1}, 1284 \mathrm{~cm}^{-1}, 1163 \mathrm{~cm}^{-1}, 1061 \mathrm{~cm}^{-1}$, and $898 \mathrm{~cm}^{-1}$ that are attributed to bending vibration of - $\mathrm{C} 6-\mathrm{CH} 2$ group [59], C-C ring starching mode, and $\mathrm{C}-\mathrm{O}-\mathrm{C}$ pyranose ring starching mode of nanocellulose [52], respectively. From Figure 2, hybrid samples showed the combination of distinctive bends compared to pure GO and CNF spectra, for instance, vibration mode of $v(\mathrm{OH}), v(\mathrm{C}-\mathrm{O})$, and $v(\mathrm{C}-\mathrm{O}-\mathrm{C})$ of graphene origin and characteristic bend of cellulose position at 1430 and $876 \mathrm{~cm}^{-1}$. Interestingly, the peak associated with $v(\mathrm{COOH})$ at 1715 and $1732 \mathrm{~cm}^{-1}$ in the spectra of GO and $\mathrm{CNF}$, respectively, disappeared after hydrothermal treatment in both spectra of control and hybrid samples. Compared to GO, control sample exhibits new prominent peaks of carboxylic $(\mathrm{C}=\mathrm{O})$ starching $\left(1663 \mathrm{~cm}^{-1}\right)$ and $\mathrm{N}-\mathrm{H}$ bending $\left(1618 \mathrm{~cm}^{-1}\right)$ of amide I and considerable shift in $\mathrm{OH}$ starching (3422-3445 $\left.\mathrm{cm}^{-1}\right)$, while hybrid samples display lower shift in band related to $\mathrm{O}-\mathrm{H}$ starching $\left(3432-3438 \mathrm{~cm}^{-1}\right)$ and considerable shift in $\mathrm{N}-\mathrm{H}$ bending $\left(1572-1633 \mathrm{~cm}^{-1}\right)$ and $\mathrm{C}-\mathrm{O}-$ $\mathrm{C}$ and C-O starching (at 1076-1087 $\mathrm{cm}^{-1}$ ) after hydrothermal treatment. This phenomenon is suggesting that the presence of carbamide plays important role for the self-assembly of monolithic material and promotes chemical interaction force, presumably, hydrogen bonding [60] as evident from shifting of these functional groups, since we failed to obtain 


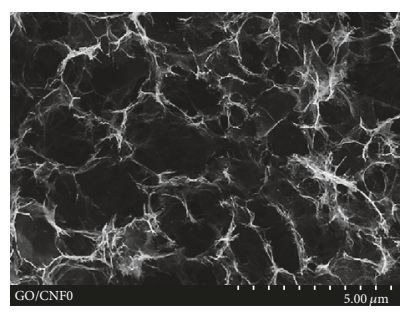

(a)

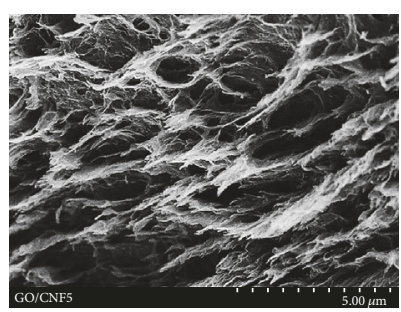

(e)

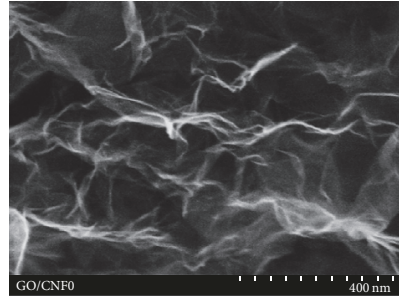

(b)

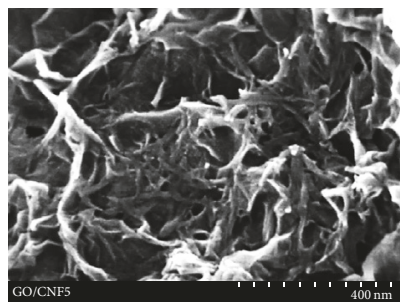

(f)

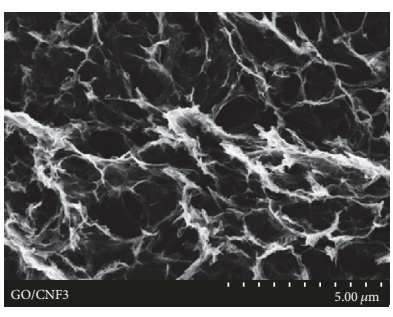

(c)

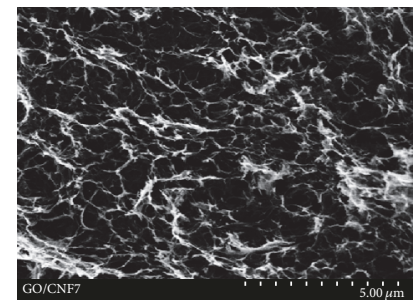

(g)

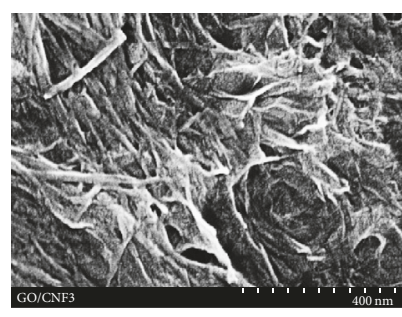

(d)

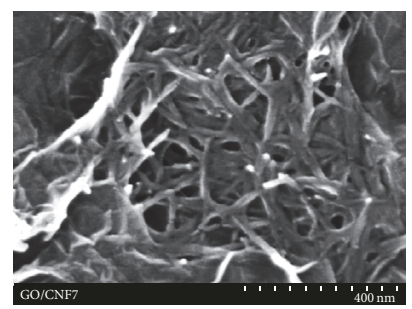

(h)

Figure 1: SEM images of control (a-b), GO/CNF3 (c-d), GO/CNF5 (e-f), and GO/CNF7 (g-h) monolith.
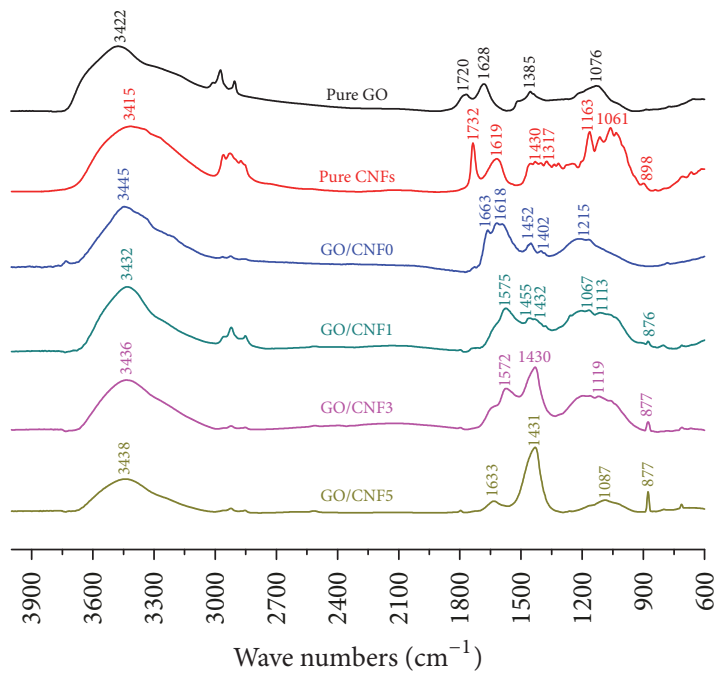

FIGURE 2: FTIR spectra of GO, CNFs, and hybrid monolith.

3D monolith in the absence of carbamide (digital image not presented here). However, nature of hydrogen bonding between bare graphene sheets and/or between GO sheets and nanocellulose fibers follows different routes. Compared to control sample, hybrid spectrum exhibits downshifting at starching vibration of hydroxyl $(\mathrm{OH})$, carboxyl (C-O, C-O$\mathrm{C}$ ), and $\mathrm{N}-\mathrm{H}$ bending vibration mode. This shift can be seen as synergy effect of strong hydrogen bonding between graphene sheets and nanocellulose fibers similar to previous reports [60], thus greatly influencing the microstructure morphology and mechanical and thermal properties of hybrid material (see Figures 1, 5, and 6). It can be inferred that strong chemical interaction between GO and CNFs is predominantly influenced by $\mathrm{C}=\mathrm{O} \cdots \mathrm{HO}$ and $\mathrm{N}-\mathrm{H} \cdots \mathrm{OH}$ type hydrogen bonding during the hydrothermal treatment. Moreover, shrinkage in $\mathrm{OH}$ starching was due to deoxygenation of graphene.
3.3. Elemental Analysis. To further expose the surface properties difference, $\mathrm{C} / \mathrm{O}$ and $\mathrm{C} / \mathrm{N}$ surface atomic ratio of samples was investigated by elemental analysis (Table S1). The $\mathrm{C} / \mathrm{O}$ ratio of hybrid samples GO/CNF3, GO/CNF5, and $\mathrm{GO} / \mathrm{CNF} 7$ showed gradual decrease in $\mathrm{C} / \mathrm{O}$ atomic ratio $(3.46,2.94$, and 2.79 , resp.) that is much lower then GO/CNF0 (5.20) but higher than starting material of GO (0.94). This can be ascribed to partial reduction of oxygen containing groups due to incorporated quantity of nanocellulose fibers, accordingly regulating the compatibility of hybrid material with aqueous medium compared to more hydrophobic counterparts ( $\mathrm{GO} / \mathrm{CNF} 0)$. Interestingly, The $\mathrm{C} / \mathrm{N}$ ratio of hybrid exhibits gradual increase compared to GO/CNF0 (11.48) and GO $(\leq 0.05)$ at the same time. It means, in case of $\mathrm{GO} / \mathrm{CNF} 0$, reduction process accompanied by incorporation of amine groups between GO sheets while breaking down carbamide during the hydrothermal treatment. On the other hand, it behaves like cross-linker between the GO sheets and cellulose nanofibers chains presumably through a reaction of amide or hydrogen bonding, also confirmed by FTIR and Raman analysis. So we can safely conclude that, by suitable incorporation of nanocellulose with graphene, one can tune the surface property and hydrophobic nature of reduced graphene.

3.4. Raman Spectra Analysis. The comparison of Raman spectra has been presented in Figure 3. It can be observed that samples such as GO/CNF3, CNF5, and CNF7 display gradual increase in the intensity of $\mathrm{D}$ band $(\mathrm{C}-\mathrm{C})$ compared to graphene oxide and GO/CNF0 samples (Figure 3 and Table S2). These results are again confirming that, during the hydrothermal treatment, chemical bonding took place between the GO sheets and adjacent CNFs similar to previous reported results [61]. To further confirm the role of CNFs as multifunctional agent we also compare the intensity ratio $\left(I_{\mathrm{D}} / I_{\mathrm{G}}\right)$ of $\mathrm{GO}$ with hybrid monolith $(\mathrm{GO} / \mathrm{CNF} 3, \mathrm{GO} / \mathrm{CNF} 5$, 


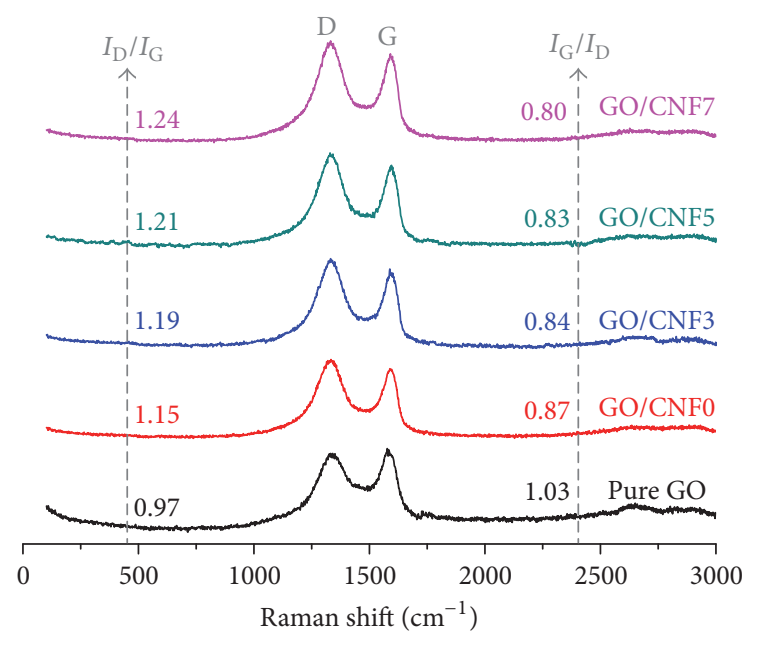

FIGURE 3: Raman spectra of pure GO, control, and hybrid monolith.

and $\mathrm{GO} / \mathrm{CNF} 7)$. As expected, there is gradual increase in intensity ratio (0.97-1.24) of hybrid with increasing amount of CNFs. This change indicated that the average $\mathrm{sp}^{3}$ hybridized (D) domains of graphene were increased upon compounding of CNFs thereby introducing more C-C atom due to substitution of hydroxyl group with amine that would reduce GO to RGO. Moreover, hybrid surface also shows little increase in $\mathrm{sp}^{2}$ hybridized ( $\mathrm{G}$ band, $\mathrm{C}=\mathrm{C}$ ) domains in amplitude but smaller number (0.80) compared to GO/CNF0 (0.87) (Figure 3). This finding was in good agreement with strong hydrogen bonding interaction between nanocellulose and graphene sheets (see FTIR).

3.5. XRD Analysis. The XRD structural patterns of graphene oxide, carboxylated cellulose nanofibers (CNFs), and hybrid samples with different mass ratio of nanocellulose to graphene have been shown in Figure S3. The feature diffraction peak of graphene oxide at $10.7^{\circ}$ corresponding to (001) showed remarkable increase in interlay space $(8.2 \AA)$, due to existence of oxygenated functional groups and trapped water molecules on both sides of sheet surface [33]. CNFs pattern exhibits feature diffraction peak of cellulose I structure at $16.5^{\circ}(110), 22.7^{\circ}(200)$, and $34.6^{\circ}(004)$, respectively. The broad peak at $22.7^{\circ}$ confirmed two feature aspects of carboxylated cellulose nanofibers [55]: (1) CFNs retain the original fiber form after TEMPO induced selective oxidation and (2) the introduced reactive carboxyl moieties were not formed inside the crystallites. The structural composition of control (GO/CNF0) and cellulose compounding hybrids showed the disappearance of representative diffraction peak of graphene oxide at $10.7^{\circ}$ (002) and appearance of new broad peak at $24.5^{\circ}(d$-spacing $=3.6-3.9 \AA)$ at the same time (Figure S3). This phenomenon can be explained as follows: the interlay space of GO was reduced due to destruction of regular stacks of graphene oxide. Moreover, except GO/CNF0, new diffraction peak at $22.5^{\circ}$ (002) originating from cellulose nanofibers crystallites was observed in all CNFs compounding samples which again confirms the successful incorporation of nanocellulose with graphene through strong amide/hydrogen bonding between CNFs with GO sheets, with gradual increase in cellulose originating peak confirming the crystalline nature of hybrid.

3.6. Thermal Stability and Chemical Composition Analysis. Thermal stability and chemical composition of samples were examined by TGA curve analysis. TGA curves of GO and CNFs showed minor weight loss at $\cong 100^{\circ} \mathrm{C}$ (Figures S4 and S5), which was attributed to dehydration of adsorbed water molecules [43]. After that, both samples exhibited one-step degradation behavior with onset temperature of $198^{\circ} \mathrm{C}$ and $227^{\circ} \mathrm{C}$ and major mass loss of $20 \%$ and $59 \%$ due to decomposition of reactive oxygen containing groups from the backbones of GO and CNFs, respectively. This result indicates that prepared graphene oxide and nanocellulose fibers were well oxidized and contain lot of oxygen containing groups. From Figure S5, hybrid samples showed gradual improvement in onset degradation temperature $\left(288^{\circ} \mathrm{C}, 295^{\circ} \mathrm{C}\right.$, and $\left.319^{\circ} \mathrm{C}\right)$ in order to increase nanocellulose content. The significantly higher thermal stability compared to GO $\left(198^{\circ} \mathrm{C}\right)$, CNFs $\left(227^{\circ} \mathrm{C}\right)$, and $\mathrm{GO} / \mathrm{CNF} 0\left(162^{\circ} \mathrm{C}\right)$ indicating the formation of more stable oxygen containing functional groups as a result of strong bonding interaction between GO and cellulose molecules [57]. It is interesting to note that, by increasing the content of CNFs $(12 \%, 20 \%$, and $28 \% \mathrm{w} / \mathrm{w})$, hybrid material showed an incremental mass loss of 6.6\% (GO/CNF3), $10.73 \%$ (GO/CNF5), and $15.63 \%$ (GO/CNF7) as well (Figure S5). This finding is indicating that about $50 \%$ oxygen functional groups of cellulose nanofibers were utilized for boding interaction, while remaining $50 \%$ had participated in enhancing surface functionality of hybrid monolith, also confirmed by elemental analysis (Table S1). Furthermore, the weight loss trends also suggest that maximum $15.63 \%$ mass of CNFs could successfully be incorporated with $\mathrm{GO}$ via self-assembly of hybrid monolith.

3.7. Compression Stress and Strain Analysis. For industrial point of view mechanical stability of monolith material is very important in order to overcome recontamination issue and reduced operating cost. Compression behaviors of control and nanocellulose incorporated hybrid monolith (GO/CNF7) are shown in Figure 4(a). Hybrid monolith displays excellent mechanical property and can maintain its original structure up to $380 \mathrm{Kpa}$ at $80 \%$ strain, which was five times higher than that of GO/CNF0 $(75 \mathrm{KPa})$ and about threefold higher than reported graphene sponge $(140 \mathrm{Kpa})$ [31]. This dramatic increase is attributed to synergy improvement in microstructure due to strong interaction between $\mathrm{GO}$ and nanocellulose fibers. To further expose the structural ability of hybrid monolith compression stress-strain cycling has been presented in Figure 4(b). It is worth noting that hybrid sample could preserve the structure $(25 \mathrm{KPa}$ at $20 \%$ strain) and recover to its original shape in all five consecutive cycles. Based on mentioned surface functionality and mechanical and elastic property of hybrid monolith, we can safely conclude that candidate monolithic material would be promising to avoid risk of recontamination during adsorption-desorption cycles, also confirmed by adsorptiondesorption cycles of model dye (Video S1). 


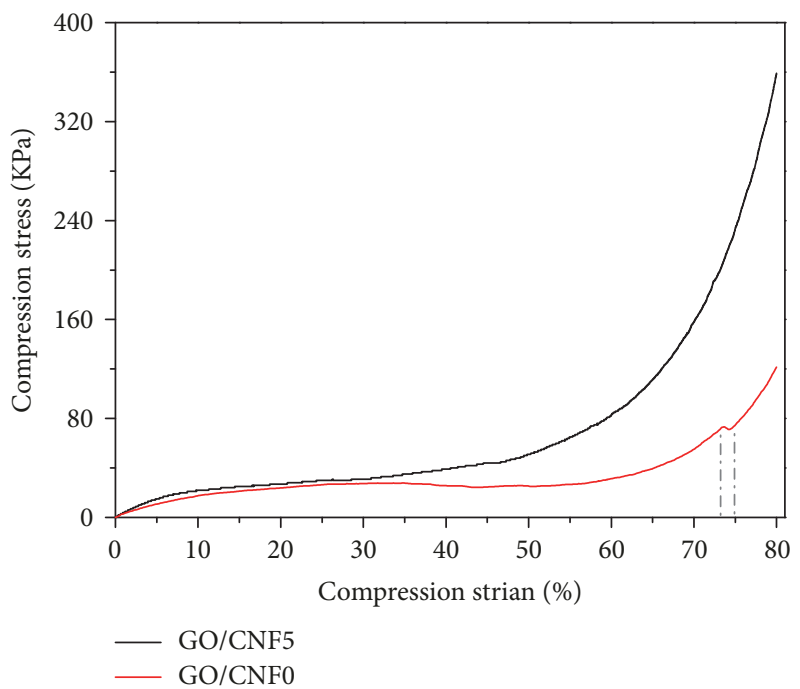

(a)

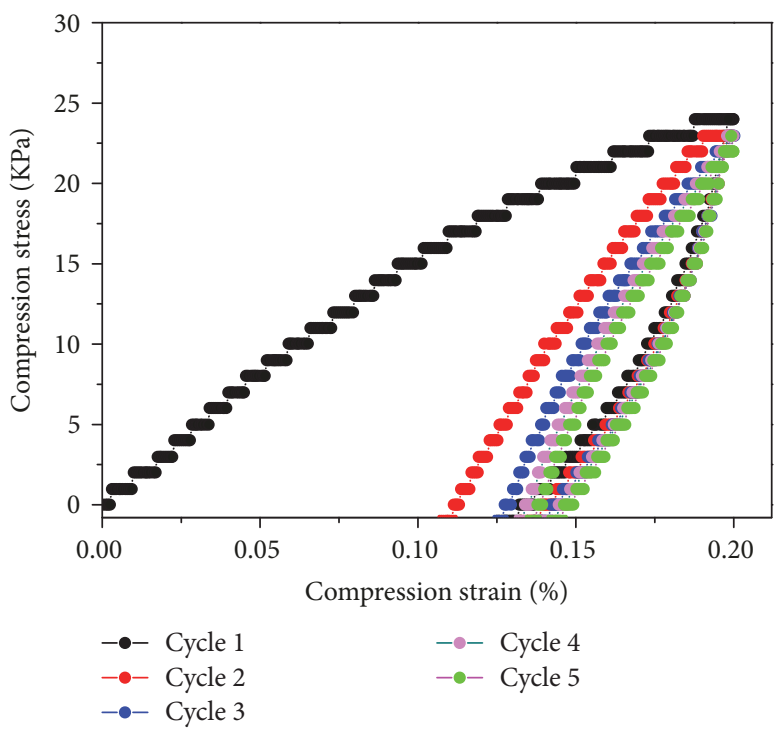

(b)

FIGURE 4: Mechanical property of control and hybrid monolith (a) and cyclic stress and strain performance of GO/CNF7 (b).

3.8. Dye Adsorption Studies. Adsorption isotherm studies can provide the information about the surface property and adsorption mechanism for any adsorbent. For this, methylene blue $(\mathrm{MB})$ dye was selected to examine the surface property difference between GO/CNF0, GO/CNF3, GO/CNF5, and $\mathrm{GO} / \mathrm{CNF} 7$. It is worth noting that nanocellulose compounded samples showed excellent adsorption capacity compared to control one (Figure 5(a)) and display linear relationship with initial $\mathrm{MB}$ dye concentration in the beginning and then attain saturation at higher concentration range. To further demonstrate the adsorption performance of dye, the logarithmic expression of same dye concentration was plotted against $\mathrm{MB}$ removal percentage. From Figure 5(b), it can be comprehended that, except GO/CNF0 (Figure 5(b)), all hybrid samples exhibited complete removal ability (100\%) for $\mathrm{MB}$ dye in very low to moderate concentration range. This finding indicates that hybrid samples have more available active sites to interact with dye molecules near low to moderate concentration region and vice versa. To confirm the reason behind the different adsorption mechanisms of samples, we investigated their zeta potential measurement. From Table S3, it can be seen that all samples have negative surface charges and their average values were found to be in the following order: GO/CNF7 > GO/CNF5 > GO/CNF3 $>\mathrm{GO} / \mathrm{CNF} 0$, respectively. It means the higher the surface negative charge, the higher the rate of adsorption and the superior the performance for positively charged MB dye. For that reason, we can conclude that nanocellulose compounded samples have strong interaction for $\mathrm{MB}$ molecules compared to $\mathrm{GO} / \mathrm{CNF} 0$ and follow different mechanism.

In order to quantitatively examine the adsorption capacity of GO/CNF3, GO/CNF5, and GO/CNF7, adsorption isotherm data were fitted to linearized form of Freundlich isotherm (Equation S3), Langmuir isotherm (Equation S4), and Temkin isotherm model (Equation S5) (see ESI Section 2). Statistical parameters of model were extracted by fitting the equation of linear regression on experimental data and summarized in Table S5. Form Figure S6, Freundlich and Temkin model showed deviation in linearity compared to Langmuir isotherm model for all hybrid samples (Figure 5(c)). Good linearity and higher value of regression coefficient $\left(r^{2}=0.99\right)$ for Langmuir model confirm that the $\mathrm{MB}$ adsorption follows monolayer type adsorption process and is found to be in the following order: Langmuir model $>$ Freundlich model $>$ Temkin model (Table 1). The $1 / n$ values of Freundlich model lie between 0 and 1, which also confirm the normal Langmuir isotherm. Moreover, samples also exhibit increasing KL value with respect to incorporated quantity of $\mathrm{CNF}$, which reflect the strong affinity for $\mathrm{MB}$ molecules via strong electrostatic interaction. Based on Langmuir model, the maximum adsorption capacities of GO/CNF3, GO/CNF5, and GO/CNF7 adsorbent were in the following order: $151.51 \mathrm{mgg}^{-1}<204.08 \mathrm{mgg}^{-1}<$ $227.27 \mathrm{mg} \mathrm{g}^{-1}$, respectively. This synergy improvement was inevitable due to alteration in microstructure (see Figure 1) and surface chemistry. For further comprehension, we also measured the specific surface area (SSA) of hybrid adsorbent (Table S3). The specific surface areas of samples were found to be in the following order: GO/CNF3 < GO/CNF5 $<\mathrm{GO} / \mathrm{CNF} 7$, with corresponding value of $324.28 \mathrm{~m}^{2} \mathrm{~g}^{-1}$, $436.79 \mathrm{~m}^{2} \mathrm{~g}^{-1}$, and $486.43 \mathrm{~m}^{2} \mathrm{~g}^{-1}$, respectively, which can be a result of increasing space between adjacent graphene sheets due to well-dispersed nanocellulose fibers. Consequently, sample with large surface area showed higher $\mathrm{MB}$ adsorption due to more available surfaces. However, higher content of CNFs showed little improvement in dye adsorption capacity and specific surface area, which could be explained as follows: higher incorporation of CNFs leads to aggregation into 


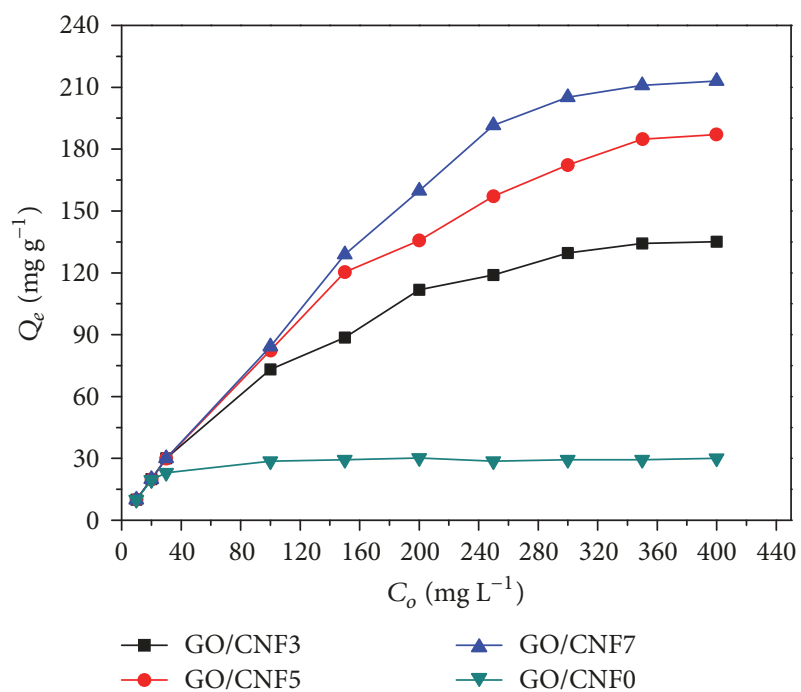

(a)

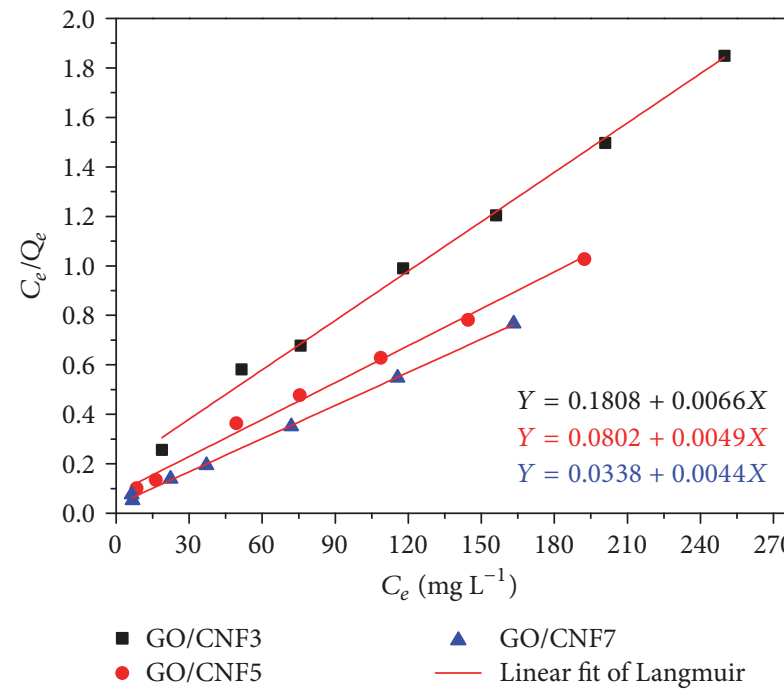

(c)

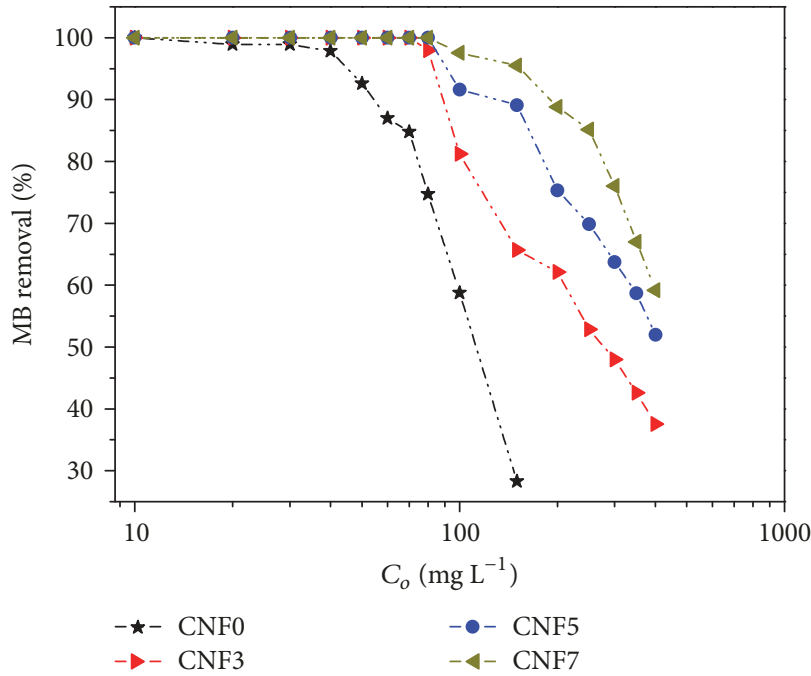

(b)

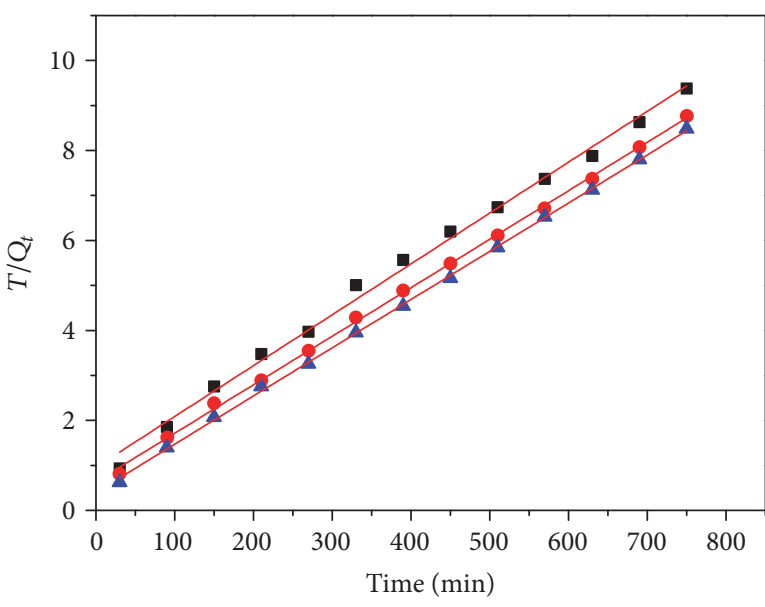

- $\mathrm{GO} / \mathrm{CNF} 3$

- $\mathrm{GO} / \mathrm{CNF} 5$
- GO/CNF7

— Linear fit of $T / Q_{t}$

(d)

Figure 5: Plots of equilibrium adsorption quantity (a), \% removal (b), Langmuir isotherm model (c), and pseudo-second-order kinetics (d) for MB dye.

TABLE 1: Equilibrium adsorption isotherm parameters of hybrid monolith for MB dye.

\begin{tabular}{|c|c|c|c|c|c|c|c|c|c|c|}
\hline \multirow[b]{2}{*}{ Sample (ID) } & \multicolumn{3}{|c|}{ Freundlich model } & \multicolumn{3}{|c|}{ Langmuir model } & \multicolumn{4}{|c|}{ Temkin model } \\
\hline & $1 / n$ & $\begin{array}{c}K_{F} \\
\left(\mathrm{~L} \mathrm{mg}^{-1}\right)\end{array}$ & $R^{2}$ & $\begin{array}{c}Q_{\max .} \\
\left(\mathrm{mg} \mathrm{g}^{-1}\right)\end{array}$ & $\begin{array}{c}K_{L} \\
\left(\mathrm{~L} \mathrm{mg}^{-1}\right)\end{array}$ & $R^{2}$ & $B_{T}$ & $\begin{array}{c}K_{T} \\
\left(\mathrm{~L} \mathrm{mg}^{-1}\right)\end{array}$ & $\begin{array}{c}b_{T} \\
\left(\mathrm{~J} \mathrm{~mol}^{-1}\right)\end{array}$ & $R^{2}$ \\
\hline $\mathrm{GO} / \mathrm{CNF} 3$ & 0.256 & 34.329 & 0.954 & 151.51 & 0.0365 & 0.995 & 26.145 & 0.798 & 96.352 & 0.956 \\
\hline GO/CNF5 & 0.246 & 53.357 & 0.935 & 204.08 & 0.0608 & 0.994 & 32.413 & 1.776 & 77.720 & 0.962 \\
\hline GO/CNF7 & 0.238 & 70.316 & 0.771 & 227.27 & 0.1302 & 0.998 & 35.614 & 3.568 & 70.734 & 0.869 \\
\hline
\end{tabular}

Conditions: $\mathrm{pH}=6.5 ; \mathrm{T}=303 \mathrm{~K}$; dosage $=1.11 \mathrm{~g} \mathrm{~L}^{-1}$; contact time $=24 \mathrm{~h} ; \mathrm{Co}=100-350 @ 50 \mathrm{mg} \mathrm{L}^{-1}$.

pore orifice thus reducing the full surface area coverage of hybrid monolith for dye molecules. Based on Table S4, candidate hybrid adsorbent showed notable adsorption performance compared with other reported work such as high cost carbon nanotube (CNT) [13], graphene/CNT monolith [33], MWCNT aerogel [51], graphene by toxic hydrazine
[27], graphene/luffa sponge [12], thiourea assisted graphene sponge [31], polydopamine microspheres [23], polyaniline hydrogel [26], magnetic particle based graphene composite $[9,19,34-38]$, graphene coated biochar [40], and graphene oxide/calcium alginate [14]; for more comparison see Table S4. 


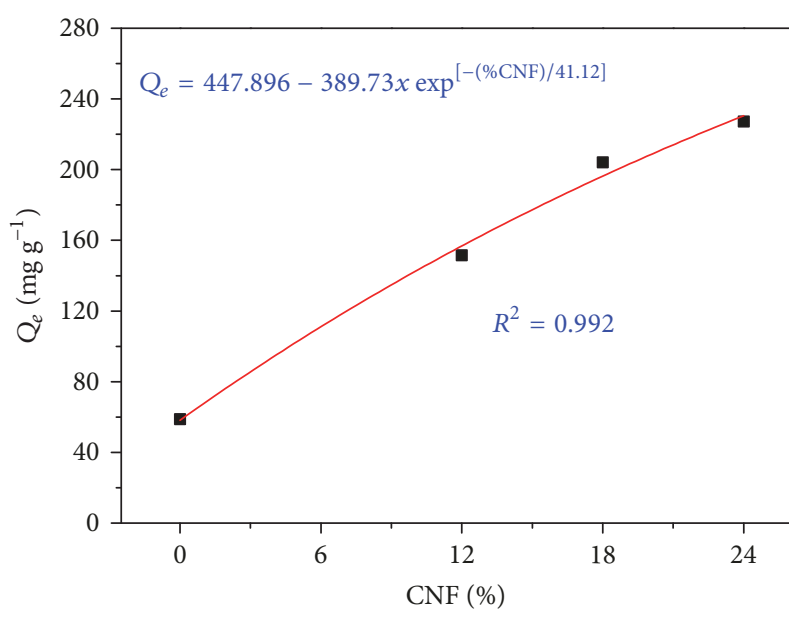

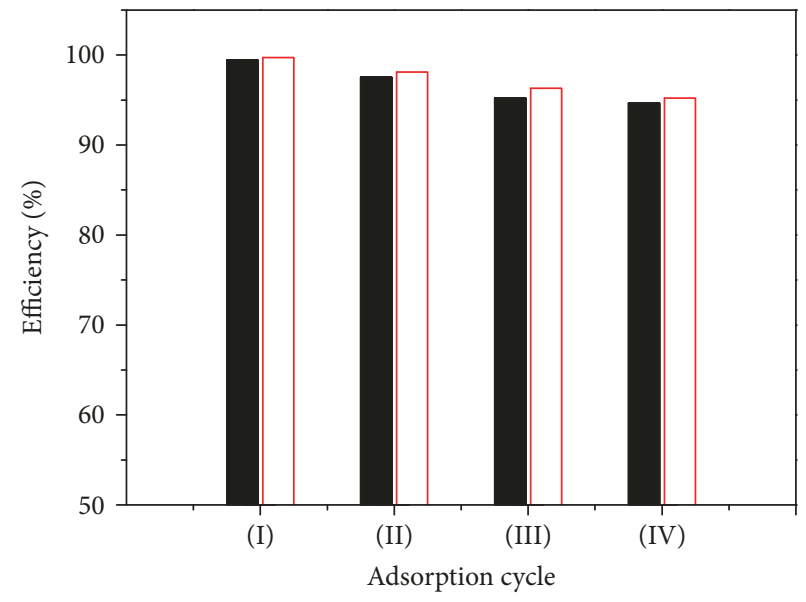

$\mathrm{GO} / \mathrm{CNF} 5$ $\mathrm{GO} / \mathrm{CNF} 7$

FIGURE 6: Effect of \% nanocellulose loading (a) and recycling performance (b) for hybrid monolith.

TABLE 2: Adsorption kinetics parameters of hybrid monolith for MB dye.

\begin{tabular}{lccccccc}
\hline & \multicolumn{3}{c}{ Pseudo-first-order model } & \multicolumn{3}{c}{ Pseudo-second-order model } \\
Sample (ID) & $\begin{array}{c}k_{1} \\
\left(\mathrm{~min}^{-1}\right)\end{array}$ & $\begin{array}{c}Q_{e, \text { cal. }} \\
\left(\mathrm{mg} \mathrm{g}^{-1}\right)\end{array}$ & $R^{2}$ & $\begin{array}{c}k_{2} \\
\left(\mathrm{~g} \mathrm{mg}^{-1} \mathrm{~min}^{-1}\right)\end{array}$ & $\begin{array}{c}Q_{e, \text { cal. }} \\
\left(\mathrm{mg} \mathrm{g}^{-1}\right)\end{array}$ & $R^{2}$ & $Q_{e \text { Exp. }}$ \\
\hline GO/CNF3 & 0.003961 & 5.68 & 0.964 & 0.00013 & 88.41 & 0.995 & 83.4 \\
GO/CNF5 & 0.004353 & 5.42 & 0.984 & 0.00018 & 92.76 & 0.999 & 88.94 \\
GO/CNF7 & 0.005389 & 4.95 & 0.968 & 0.00029 & 93.28 & 0.999 \\
\hline
\end{tabular}

Conditions: $\mathrm{Co}=100 \mathrm{mg} \mathrm{L}^{-1} ; \mathrm{pH}=6.56 ; \mathrm{T}=303 \mathrm{~K}$; dosage $=1.11 \mathrm{~g} \mathrm{~L}^{-1}$.

Generally, adsorption kinetics study predicts the rate of adsorption and nature of interaction for any adsorption process. In this study, pseudo-first order and pseudosecond order were selected to understand the adsorption kinetics of hybrid adsorbent (GO/CNF3, GO/CNF5, and GO/CNF7). Pseudo-first order model considers that rate of adsorption process is proportional to number of unoccupied sites, while pseudo-second-order model suggests that rate of adsorption process is proportional to square of number of unoccupied sites. The linearized forms of pseudo-first order (Equation S1) and pseudo-second order (Equation S2) along with model parameter were incorporated in ESI Section 2. Kinetic parameters were obtained by plotting the linear graph of $\log \left(Q_{e}-Q_{t}\right)$ versus $t$ (Figure S6) and $t / Q_{t}$ versus $t$ (Figure 5(c)), respectively. From Table 2, pseudofirst-order parameters exhibit poor relationship with lower coefficient of correlation $\left(r^{2}\right)$ and significant deviation in calculated and experimental $Q_{e}$ values for all hybrid samples (Figure S6a and Table 2). This finding confirms that the adsorption of $\mathrm{MB}$ dye did not follow the pseudo-first-order kinetic model. The linear plot of pseudo-second-order model showed excellent fit with higher correlation coefficient $r^{2} \geq$ 0.99 for all hybrid material (Figure 5(c) and Table 2). Good linear relationship and very close calculated and measured values of $Q_{e}$ approve that adsorption process of $\mathrm{MB}$ dye onto hybrid samples was mainly governed by pseudo-secondorder model and chemisorption type process in nature. Interestingly, upon compounding of nanocellulose fibers, adsorption rate constant $\left(k_{2}\right)$ and performance of hybrid samples (GO/CNF3, GO/CNF5, and GO/CNF7) also showed gradual improvement (Table 2). This can be attributed to alteration in surface property and charge density of material and agree with the results of zeta potential measurement (Table S3).

3.9. Effect of Nanocellulose Quantity. As mentioned above, the incorporation of different content of nanocellulose brought notable change in adsorption efficiency and rate of hybrid adsorbent compared to control sample (Tables 1 and 2). To further clarify this, statistical relationship between equilibrium $\mathrm{MB}$ adsorption quantity and effect of \% CNFs loading was established in Figure 6(a). It can be observed that dye adsorption quantity followed exponential relationship of $Q_{e}=30 * \exp ^{[-(\mathrm{CNFs} / 22.72)]}$ with incorporated nanocellulose quantity. Good applicability of this relationship $\left(r^{2}=0.994\right)$ confirms that nanocellulose loading imparts remarkable change in developed surface such as higher oxygen containing sites, surface roughness, and specific surface area which greatly benefits capturing more MB molecules in solution (see Tables S1 and S3 and SEM images). Based on this, we 
can safely conclude that interaction of MB dye with control (hydrophobic) and hybrid (hydrophilic) surface followed different mechanism. This means electrostatic interaction was more dominant than hydrophobic type $\pi-\pi$ interaction in hybrid adsorbent.

3.10. Adsorption-Desorption Studies. From the industrial point of view, the structural stability and recycling study is very important to reduction of operating cost and practical qualification of adsorbent materials. In order to examine the adsorption and regeneration performance, first hybrid GO/CNF5 adsorbent was put into MB aqueous solution and dye concentration was measured every $30 \mathrm{~min}$ by UV-VIS spectra analysis. It is worth noting that $100 \%$ removal was achieved within $150 \mathrm{~min}$ (Figures S7 and S8). After removal, spent adsorbent was placed in the mixture of HCL (2\%) and ethanol $(1: 1 \mathrm{v} / \mathrm{v})$ under mild stirring. Based on Figure S8 and Video S1, MB dye can easily be released from adsorbent without losing any detachable particle in the solution which successfully qualifies the adsorption and regeneration ability of adsorbent with zero release of nanohazard into treated water. To further investigate the recycling ability of hybrid adsorbents, adsorption-desorption process was performed for four consecutive cycles. It is worth noting that, with the exception of little decline in efficiency $(\cong 6 \%)$, hybrid adsorbent showed still higher adsorption capacity for four consecutive cycles (Figure 6(b)). Based on excellent adsorption-desorption performances and structural stability, $\mathrm{GO} / \mathrm{CNF}$ adsorbent can be promising candidate for water purification industry.

\section{Conclusion}

Hybrid monolith of graphene oxide and nanocellulose fibers with robust interconnected network was fabricated via ecofriendly self-assembly method. Pore morphology and surface chemistry (such as hydrophilicity and surface charge density) of monolith were improved. Specific surface area and mechanical property were enhanced about four- and fivefold, respectively, compared to unmodified monolith. It is proposed that strong chemical interaction, mainly hydrogen bonding, was primary driving force for the formation of hybrid monolith. Maximum $15.63 \%$ w/w grafting of nanocellulose was achieved. Incorporation of nanocellulose exhibits synergy improvement in dye adsorption performance. Complete removal was achieved for trace to moderate quantity of dye. Adsorption process followed monolayer type Langmuir isotherm model. High surface area coupled with electrostatic interaction was suggested for dye adsorption mechanisms. Adsorption capacity followed exponential relationship with incorporated nanocellulose content. Hybrid monolith showed simple and cost-effective recollection and recycling performance. Further application of hybrid material is underway in our lab.

\section{Additional Points}

Associated Content. Supporting information includes detailed description for preparation of TEMPO-oxidized cellulose and batch adsorption studies: FTIR spectra of carboxylated nanocellulose, macroscopic images of hybrid monolith, Xray pattern analysis, TGA curve analysis, digital images of dye adsorption, dye desorption video, adsorption isotherm models descriptions and plots, tables of elemental analysis, Raman spectra analysis, zeta potential analysis, specific surface area measurement, adsorption isotherm, kinetics parameters, and comparison of performance for $\mathrm{MB}$ adsorption capacity with reported work.

\section{Conflicts of Interest}

The authors declare no conflicts of interest.

\section{Acknowledgments}

This work was financially supported by the National Natural Science Foundation of China (U1660107), Textile Light Application Basic Research of China (J201503), and Specialized Research Fund for the Doctoral Program of Senior Education of China (Grant no. 20130075110006).

\section{Supplementary Materials}

Figure S1: FTIR spectra of cellulose and TEMPO-oxidized cellulose. Figure S2: digital images of macroscopic hybrid monolith. (a-b) Small-sized diameter and length measurement; (c-d) large-sized diameter and length measurement; (e) higher CNFs content GO/CNF10. Figure S3: X-ray diffraction spectra of GO, CNFs, and hybrid monolith. Figure S4: thermogravimetric curve (TGA) of GO and control (GO/CNF0) monolith. Figure S5: thermogravimetric curve (TGA) of CNF, control, and hybrid monolith. Figure S6: plots of Freundlich model (d), Temkin model (e), and pseudo-firstorder kinetics model of hybrid monolith (a). Figure S7: digital photograph of adsorption study of MB removal by GO/CNF7. (a) Before removal. (b) After $60 \mathrm{~min}$. (c) After $90 \mathrm{~min}$. (d) After 120 min. (e) After 150 min. Figure S8: UV-VIS spectra of MB dye removal by GO/CNF7 monolith. Video S1: MB desorption and regeneration video of GO/CNF7 monolith adsorbent. Table S1: elemental analysis of carbon (C \%), hydrogen $(\% \mathrm{H})$, nitrogen $(\% \mathrm{~N})$, and oxygen $(\% \mathrm{O})$ content of material. Table S2: Raman spectra parameters, intensity ratio, and aromatic cluster size. Table S3: zeta potential parameters of materials. Table S4: comparisons of MB adsorption capacity with other adsorbents. (Supplementary Materials)

\section{References}

[1] R. O. Alves de Lima, A. P. Bazo, D. M. F. Salvadori, C. M. Rech, D. de Palma Oliveira, and G. de Aragão Umbuzeiro, "Mutagenic and carcinogenic potential of a textile azo dye processing plant effluent that impacts a drinking water source," Mutation Research-Genetic Toxicology and Environmental Mutagenesis, vol. 626, no. 1-2, pp. 53-60, 2007.

[2] N. P. Raval, P. U. Shah, and N. K. Shah, "Adsorptive amputation of hazardous azo dye Congo red from wastewater: a critical review," Environmental Science and Pollution Research, vol. 23, no. 15, pp. 14810-14853, 2016. 
[3] E. Guibal and J. Roussy, "Coagulation and flocculation of dyecontaining solutions using a biopolymer (Chitosan)," Reactive \& Functional Polymers, vol. 67, no. 1, pp. 33-42, 2007.

[4] F. D. Castro, J. P. Bassin, and M. Dezotti, "Treatment of a simulated textile wastewater containing the Reactive Orange 16 azo dye by a combination of ozonation and moving-bed biofilm reactor: evaluating the performance, toxicity, and oxidation byproducts," Environmental Science and Pollution Research, vol. 24, no. 7, pp. 6307-6316, 2017.

[5] J. Li, A. Hussain, D. Li, M. Yang, and S. Xu, "Catalytic performance of graphene-bimetallic composite for heterogeneous oxidation of acid orange 7 from aqueous solution," Environmental Science and Pollution Research, vol. 24, no. 8, pp. 7264-7273, 2017.

[6] N. Bao, Y. Li, X.-H. Yu, J.-J. Niu, G.-L. Wu, and X.-H. Xu, "Removal of anionic azo dye from aqueous solution via an adsorption-photosensitized regeneration process on a $\mathrm{TiO} 2$ surface," Environmental Science and Pollution Research, vol. 20, no. 2, pp. 897-906, 2013.

[7] A. H. Hassani, R. Mirzayee, S. Nasseri, M. Borghei, M. Gholami, and B. Torabifar, "Nanofiltration process on dye removal from simulated textile wastewater," International Journal of Environmental Science \& Technology, vol. 5, no. 3, pp. 401-408, 2008.

[8] R. Jain, P. Sharma, and S. Sikarwar, "Kinetics and isotherm analysis of Tropaeoline 000 adsorption onto unsaturated polyester resin (UPR): a non-carbon adsorbent," Environmental Science and Pollution Research, vol. 20, no. 3, pp. 1493-1502, 2013.

[9] M. Namvari and H. Namazi, "Synthesis of magnetic citricacid-functionalized graphene oxide and its application in the removal of methylene blue from contaminated water," Polymer International, vol. 63, no. 10, pp. 1881-1888, 2014.

[10] H. Yan, X. Tao, Z. Yang et al., "Effects of the oxidation degree of graphene oxide on the adsorption of methylene blue," Journal of Hazardous Materials, vol. 268, pp. 191-198, 2014.

[11] G. Z. Kyzas, E. A. Deliyanni, and K. A. Matis, "Graphene oxide and its application as an adsorbent for wastewater treatment," Journal of Chemical Technology and Biotechnology, vol. 89, no. 2, pp. 196-205, 2014.

[12] S. Li, M. Tao, and Y. Xie, "Reduced graphene oxide modified luffa sponge as a biocomposite adsorbent for effective removal of cationic dyes from aqueous solution," Desalination and Water Treatment, vol. 57, no. 42, pp. 20049-20057, 2016.

[13] Y. Li, Q. Du, T. Liu et al., "Comparative study of methylene blue dye adsorption onto activated carbon, graphene oxide, and carbon nanotubes," Chemical Engineering Research and Design, vol. 91, no. 2, pp. 361-368, 2013.

[14] Y. Li, Q. Du, T. Liu et al., "Methylene blue adsorption on graphene oxide/calcium alginate composites," Carbohydrate Polymers, vol. 95, no. 1, pp. 501-507, 2013.

[15] F. Liu, S. Chung, G. Oh, and T. S. Seo, "Three-dimensional graphene oxide nanostructure for fast and efficient watersoluble dye removal," ACS Applied Materials \& Interfaces, vol. 4, no. 2, pp. 922-927, 2012.

[16] Y. Liu, J. Wang, Y. Zheng, and A. Wang, "Adsorption of methylene blue by kapok fiber treated by sodium chlorite optimized with response surface methodology," Chemical Engineering Journal, vol. 184, pp. 248-255, 2012.

[17] J. Praskalo, M. Kostic, A. Potthast, G. Popov, B. Pejic, and P. Skundric, "Sorption properties of TEMPO-oxidized natural and man-made cellulose fibers," Carbohydrate Polymers, vol. 77, no. 4, pp. 791-798, 2009.
[18] W. Wan, R. Zhang, W. Li et al., "Graphene-carbon nanotube aerogel as an ultra-light, compressible and recyclable highly efficient absorbent for oil and dyes," Environmental Science: Nano, vol. 3, no. 1, pp. 107-113, 2016.

[19] P. Wang, M. Cao, C. Wang, Y. Ao, J. Hou, and J. Qian, "Kinetics and thermodynamics of adsorption of methylene blue by a magnetic graphene-carbon nanotube composite," Applied Surface Science, vol. 290, pp. 116-124, 2014.

[20] J. Yener, T. Kopac, G. Dogu, and T. Dogu, "Dynamic analysis of sorption of Methylene Blue dye on granular and powdered activated carbon," Chemical Engineering Journal, vol. 144, no. 3, pp. 400-406, 2008.

[21] W. Zhang, C. Zhou, W. Zhou et al., "Fast and considerable adsorption of methylene blue dye onto graphene oxide," Bulletin of Environmental Contamination and Toxicology, vol. 87, no. 1, pp. 86-90, 2011.

[22] H. Deng, J. Lu, G. Li, G. Zhang, and X. Wang, "Adsorption of methylene blue on adsorbent materials produced from cotton stalk," Chemical Engineering Journal, vol. 172, no. 1, pp. 326-334, 2011.

[23] J. Fu, Z. Chen, M. Wang et al., "Adsorption of methylene blue by a high-efficiency adsorbent (polydopamine microspheres): kinetics, isotherm, thermodynamics and mechanism analysis," Chemical Engineering Journal, vol. 259, pp. 53-61, 2015.

[24] M. J. Iqbal and M. N. Ashiq, "Adsorption of dyes from aqueous solutions on activated charcoal," Journal of Hazardous Materials, vol. 139, no. 1, pp. 57-66, 2007.

[25] V. Vadivelan and K. Vasanth Kumar, "Equilibrium, kinetics, mechanism, and process design for the sorption of methylene blue onto rice husk," Journal of Colloid and Interface Science, vol. 286, no. 1, pp. 90-100, 2005.

[26] B. Yan, Z. Chen, L. Cai, Z. Chen, J. Fu, and Q. Xu, "Fabrication of polyaniline hydrogel: Synthesis, characterization and adsorption of methylene blue," Applied Surface Science, vol. 356, pp. 39-47, 2015.

[27] T. Liu, Y. Li, Q. Du et al., "Adsorption of methylene blue from aqueous solution by graphene," Colloids and Surfaces B: Biointerfaces, vol. 90, no. 1, pp. 197-203, 2012.

[28] G. K. Ramesha, A. V. Kumara, H. B. Muralidhara, and S. Sampath, "Graphene and graphene oxide as effective adsorbents toward anionic and cationic dyes," Journal of Colloid and Interface Science, vol. 361, no. 1, pp. 270-277, 2011.

[29] S.-T. Yang, S. Chen, Y. Chang, A. Cao, Y. Liu, and H. Wang, "Removal of methylene blue from aqueous solution by graphene oxide," Journal of Colloid and Interface Science, vol. 359, no. 1, pp. 24-29, 2011.

[30] W. Gao, "Engineered graphite oxide materials for application in water purification," ACS Applied Materials \& Interfaces, vol. 3, no. 6, pp. 1821-1826, 2011.

[31] J. Zhao, W. Ren, and H.-M. Cheng, "Graphene sponge for efficient and repeatable adsorption and desorption of water contaminations," Journal of Materials Chemistry, vol. 22, no. 38, pp. 20197-20202, 2012.

[32] J.-J. Shao, W. Lv, and Q.-H. Yang, "Self-assembly of graphene oxide at interfaces," Advanced Materials, vol. 26, no. 32, pp. 5586-5612, 2014.

[33] L. Ai and J. Jiang, "Removal of methylene blue from aqueous solution with self-assembled cylindrical graphene-carbon nanotube hybrid," Chemical Engineering Journal, vol. 192, pp. 156163, 2012. 
[34] L. Ai, C. Zhang, and Z. Chen, "Removal of methylene blue from aqueous solution by a solvothermal-synthesized graphene/magnetite composite," Journal of Hazardous Materials, vol. 192, no. 3, pp. 1515-1524, 2011.

[35] S. Bai, X. Shen, X. Zhong et al., "One-pot solvothermal preparation of magnetic reduced graphene oxide-ferrite hybrids for organic dye removal," Carbon, vol. 50, no. 6, pp. 2337-2346, 2012.

[36] L. Fan, C. Luo, X. Li, F. Lu, H. Qiu, and M. Sun, "Fabrication of novel magnetic chitosan grafted with graphene oxide to enhance adsorption properties for methyl blue," Journal of Hazardous Materials, vol. 215-216, pp. 272-279, 2012.

[37] Y. Yao, S. Miao, S. Liu, L. P. Ma, H. Sun, and S. Wang, "Synthesis, characterization, and adsorption properties of magnetic $\mathrm{Fe}_{3} \mathrm{O}_{4} @$ graphene nanocomposite," Chemical Engineering Journal, vol. 184, pp. 326-332, 2012.

[38] H. Shi, W. Li, L. Zhong, and C. Xu, "Methylene blue adsorption from aqueous solution by magnetic cellulose/graphene oxide composite: equilibrium, kinetics, and thermodynamics," Industrial \& Engineering Chemistry Research, vol. 53, no. 3, pp. 11081118, 2014.

[39] C. B. Wang, J. W. Zhou, and L. L. Chu, "Chlorine-functionalized reduced graphene oxide for methylene blue removal," RSC Advances, vol. 5, no. 65, pp. 52466-52472, 2015.

[40] M. Zhang, B. Gao, Y. Yao, Y. Xue, and M. Inyang, "Synthesis, characterization, and environmental implications of graphenecoated biochar," Science of the Total Environment, vol. 435-436, pp. 567-572, 2012.

[41] H.-P. Cong, X.-C. Ren, P. Wang, and S.-H. Yu, "Macroscopic multifunctional graphene-based hydrogels and aerogels by a metal ion induced self-assembly process," ACS Nano, vol. 6, no. 3, pp. 2693-2703, 2012.

[42] C. Li and G. Shi, "Three-dimensional graphene architectures," Nanoscale, vol. 4, no. 18, pp. 5549-5563, 2012.

[43] C.-C. Ji, M.-W. Xu, S.-J. Bao et al., "Self-assembly of threedimensional interconnected graphene-based aerogels and its application in supercapacitors," Journal of Colloid and Interface Science, vol. 407, pp. 416-424, 2013.

[44] Y. Qian, I. M. Ismail, and A. Stein, "Ultralight, highsurface-area, multifunctional graphene-based aerogels from self-assembly of graphene oxide and resol," Carbon, vol. 68, pp. 221-231, 2014.

[45] D. Cai and M. Song, "Recent advance in functionalized graphene/polymer nanocomposites," Journal of Materials Chemistry, vol. 20, no. 37, pp. 7906-7915, 2010.

[46] J. L. Vickery, A. J. Patil, and S. Mann, "Fabrication of graphene-polymer nanocomposites with higher-order threedimensional architectures," Advanced Materials, vol. 21, no. 21, pp. 2180-2184, 2009.

[47] H. Bai, C. Li, and G. Shi, "Functional composite materials based on chemically converted graphene," Advanced Materials, vol. 23, no. 9, pp. 1088-1088, 2011.

[48] Y. X. Xu, Q. Wu, Y. Q. Sun, H. Bai, and G. Q. Shi, “Threedimensional self-assembly of graphene oxide and DNA into multifunctional hydrogels," ACS Nano, vol. 4, no. 12, pp. 73587362, 2010.

[49] H. Bi, X. Xie, K. Yin et al., "Graphene: spongy graphene as a highly efficient and recyclable sorbent for oils and organic solvents," Advanced Functional Materials, vol. 22, no. 21, pp. 4401-4401, 2012.
[50] J. Wang, Z. Shi, J. Fan, Y. Ge, J. Yin, and G. Hu, "Self-assembly of graphene into three-dimensional structures promoted by natural phenolic acids," Journal of Materials Chemistry, vol. 22, no. 42, pp. 22459-22466, 2012.

[51] N. S. Tabrizi and M. Yavari, "Methylene blue removal by carbon nanotube-based aerogels," Chemical Engineering Research and Design, vol. 94, pp. 516-523, 2015.

[52] R. Kabiri and H. Namazi, "Nanocrystalline cellulose acetate (NCCA)/graphene oxide (GO) nanocomposites with enhanced mechanical properties and barrier against water vapor," Cellulose, vol. 21, no. 4, pp. 3527-3539, 2014.

[53] A. Kafy, K. K. Sadasivuni, H.-C. Kim, A. Akther, and J. Kim, "Designing flexible energy and memory storage materials using cellulose modified graphene oxide nanocomposites," Physical Chemistry Chemical Physics, vol. 17, no. 8, pp. 5923-5931, 2015.

[54] Y. Habibi, H. Chanzy, and M. R. Vignon, "TEMPO-mediated surface oxidation of cellulose whiskers," Cellulose, vol. 13, no. 6, pp. 679-687, 2006.

[55] T. Saito and A. Isogai, "TEMPO-mediated oxidation of native cellulose. The effect of oxidation conditions on chemical and crystal structures of the water-insoluble fractions," Biomacromolecules, vol. 5, no. 5, pp. 1983-1989, 2004.

[56] M. Shimizu, H. Fukuzumi, T. Saito, and A. Isogai, "Preparation and characterization of TEMPO-oxidized cellulose nanofibrils with ammonium carboxylate groups," International Journal of Biological Macromolecules, vol. 59, pp. 99-104, 2013.

[57] A. Javadi, Q. Zheng, F. Payen et al., "Polyvinyl alcoholcellulose nanofibrils-graphene oxide hybrid organic aerogels," ACS Applied Materials \& Interfaces, vol. 5, no. 13, pp. 5969-5975, 2013.

[58] W. S. Hummers Jr. and R. E. Offeman, "Preparation of graphitic oxide," Journal of the American Chemical Society, vol. 80, no. 6, p. 1339, 1958.

[59] P. Lu and Y.-L. Hsieh, "Preparation and properties of cellulose nanocrystals: rods, spheres, and network," Carbohydrate Polymers, vol. 82, no. 2, pp. 329-336, 2010.

[60] C. Rodríguez-González, A. L. Martínez-Hernández, V. M. Castaño, O. V. Kharissova, R. S. Ruoff, and C. Velasco-Santos, "Polysaccharide nanocomposites reinforced with graphene oxide and keratin-grafted graphene oxide," Industrial \& Engineering Chemistry Research, vol. 51, no. 9, pp. 3619-3629, 2012.

[61] R. Kabiri and H. Namazi, "Surface grafting of reduced graphene oxide using nanocrystalline cellulose via click reaction," Journal of Nanoparticle Research, vol. 16, no. 7, p. 2474, 2014. 


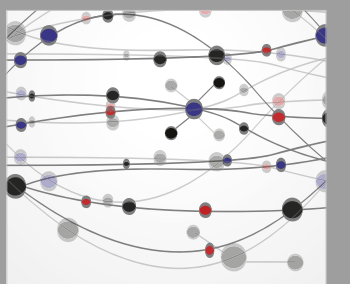

The Scientific World Journal
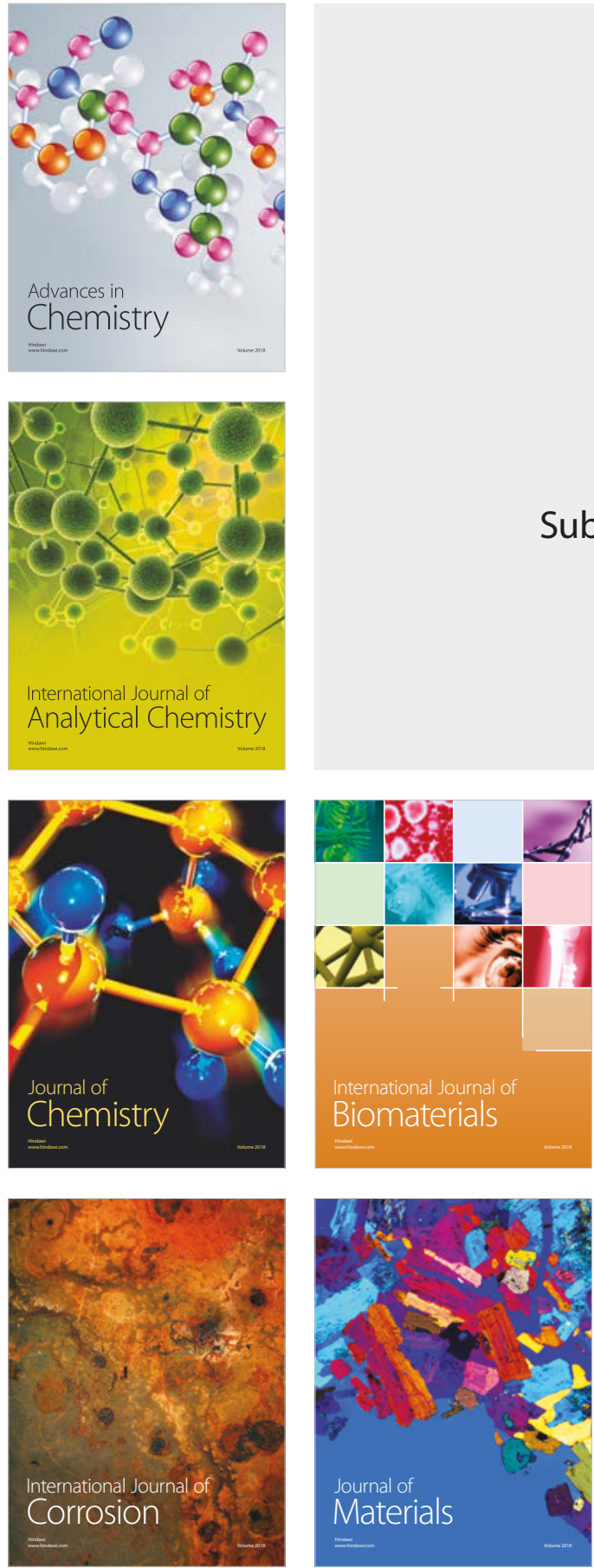

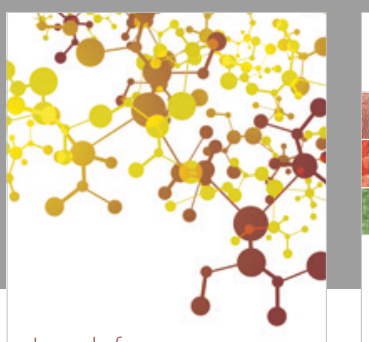

Journal of

Applied Chemistry
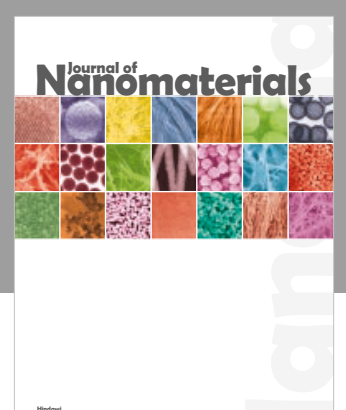

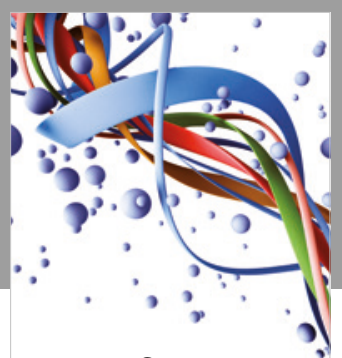

Scientifica

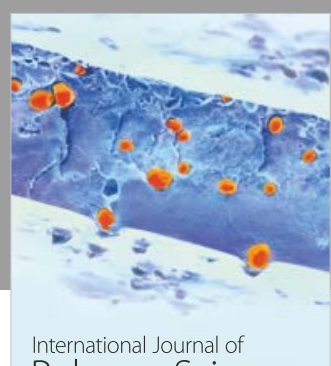

Polymer Science

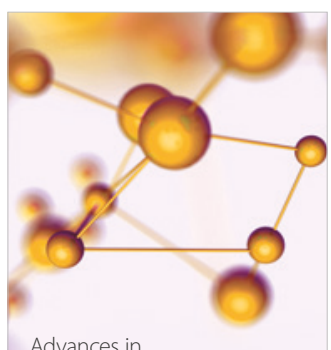

Physical Chemistry
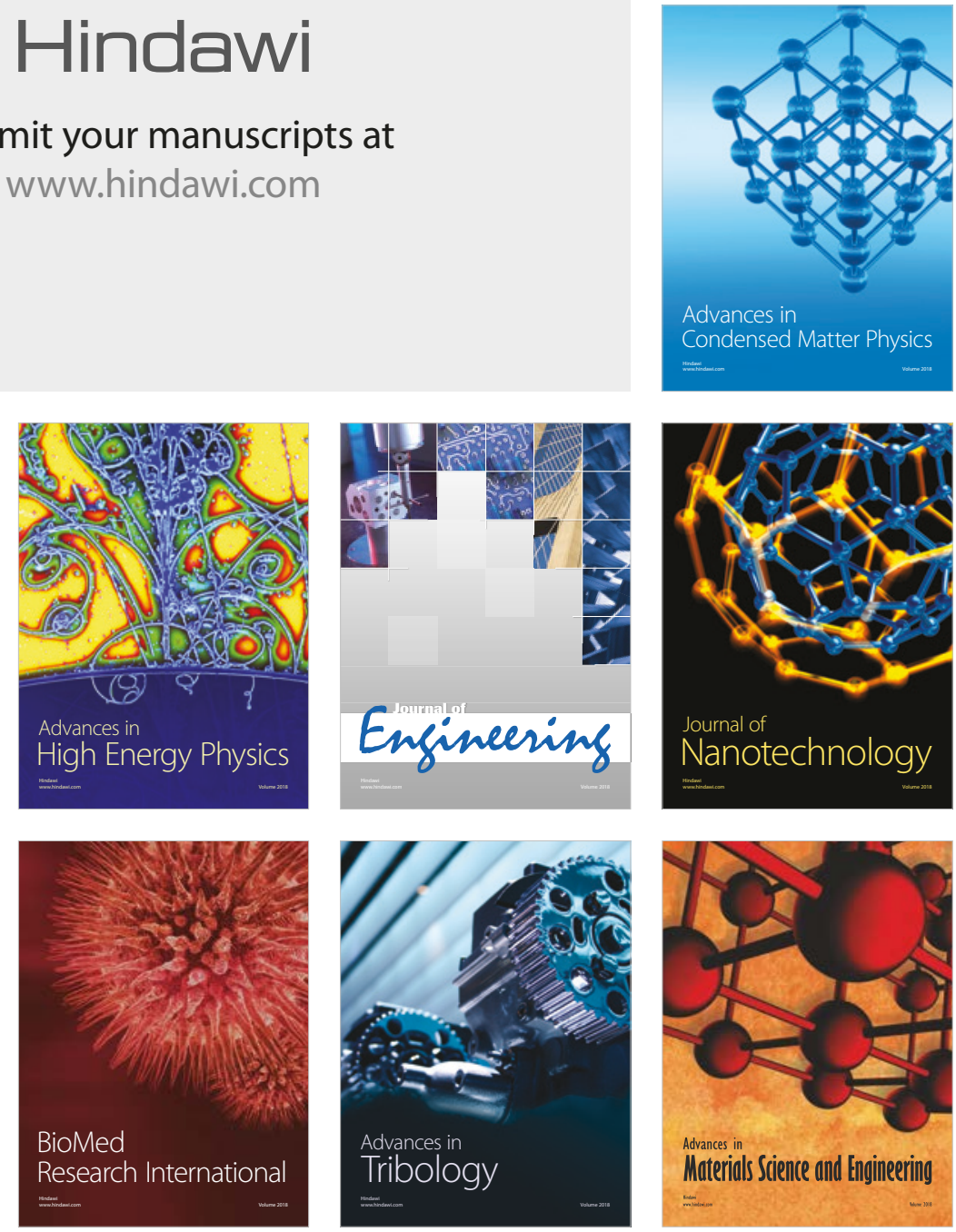